\title{
Gas adsorption and separation: tuning the channel electrostatics for $\mathrm{CO}_{2}$.
}

\author{
Charl X. Bezuidenhout, Jacopo Perego, Silvia Bracco, Piero Sozzani \\ University of Milano Bicocca, Milano, Italy \\ charl.bezuidenhout@unimib.it
}

Metal-Organic frameworks (MOFs) and porous molecular materials represent a new platform for achieving and exploring highperformance sorptive properties and gas transport. The key lies in the modular nature of these materials, which allows for tuning and functionalization towards improved gas capture.

Self-assembly of polyfunctional molecules containing multiple charges, namely, tetrahedral tetra-sulfonate anions and bi-functional linear cations, resulted in a permanently porous crystalline material in which the channels are decorated by double helices of electrostatic charges that governed the association and transport of $\mathrm{CO}_{2}$ molecules (Fig. 1). These channels electrostatically compliment the CO $\mathrm{C}_{2}$ molecules and forms strong interactions of $35 \mathrm{~kJ} \mathrm{~mol}^{-1}$, ideal for $\mathrm{CO}_{2}$ capture/release cycles.[1]

The $\mathrm{CO}_{2}$ adsorption properties were modulated for an isoreticular series of Fe-MOFs by varying the decoration of fluorine atoms within their channel (Fig. 2). A host of complementary experimental and computational techniques gives a holistic view of the host- $\mathrm{CO}_{2}$ properties towards the potential selective removal of $\mathrm{CO}_{2}$ from other gases. GCMC and DFT were employed for a detailed description of the $\mathrm{CO}_{2}$ diffusion and interactions in the porous materials. $\mathrm{CO}_{2}$-matrix adsorption enthalpies of $33 \mathrm{~kJ}$ mol-1 was accurately measured in-situ by simultaneous acquisition of micro-calorimetric and volumetric-isotherm data. Direct measurements of adsorption heats are not common and such data helps to validate mathematical models and protocols for sorption-derived adsorption enthalpies. [2]

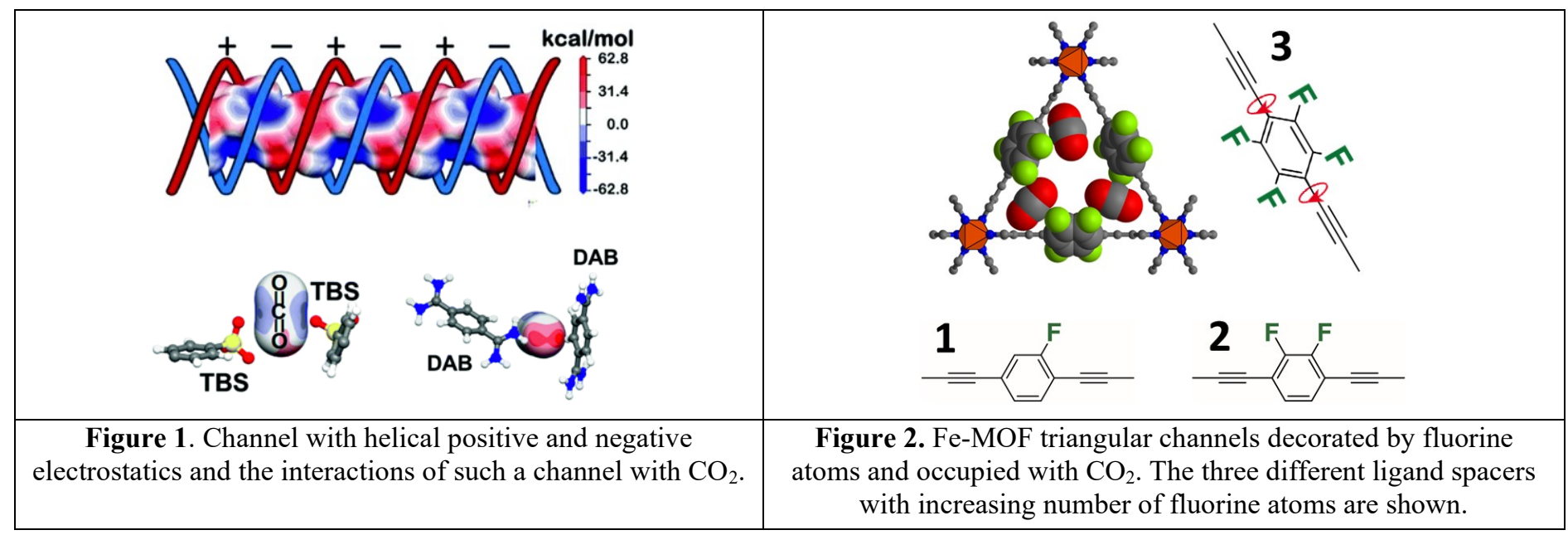

[1] Xing, G.; Bassanetti, I.; Bracco, S.; Negroni, M.; Bezuidenhout, C.; Ben, T.; Sozzani, P.; Comotti, A., Chemical Science 2019, 10 (3), $730-736$.

[2] Perego, J.; Bezuidenhout, C. X.; Pedrini, A.; Bracco, S.; Negroni, M.; Comotti, A.; Sozzani, P., Journal of Materials Chemistry A 2020, 8 (22), 11406-11413.

Keywords: Porous Materials; MOFs; Carbon dioxide; Gas Sorption

Charl X. Bezuidenhout would like to thank the INSTM Consortium/Lombardy Region and PRIN NEMO. The "Dipartimenti di Eccellenza 2018-2022" project is acknowledged for the financial support. 